\title{
Identificação do dano induzido por fadiga e cura de micro trincas em compósitos cerâmicos particulados, voltados à aplicação em pavimentos flexíveis
}

\section{(Fatigue damage induced and healing identification in particulate ceramic composites for application in flexible pavings)}

\author{
F. R. G. Nunes, C. L. da Silva, C.A.da Frota \\ Grupo de Geotecnia, Faculdade de Tecnologia, Universidade Federal do Amazonas - UFAM \\ Av. General Rodrigo Octávio Jordão Ramos, 3000, Manaus, AM, 69077-000 \\ fabiorgn@gmail.com,cleudineilopes@gmail.com,cafrota@ufam.edu.br
}

\begin{abstract}
Resumo
Um dos grandes defeitos ocorrentes no pavimento, ao longo de sua vida útil, é o trincamento por fadiga. Este problema, em geral, se inicia na base do revestimento asfáltico e se propaga para a sua superfície. A caracterização das misturas asfálticas, realizada comumente no Brasil, é baseada no número de ciclos que causam a ruptura no ensaio de fadiga. No entanto, tal número não é um parâmetro mecânico, podendo variar para diferentes condições de contorno. O objetivo do presente artigo é identificar parâmetros mecânicos, referentes ao trincamento por fadiga, do compósito asfáltico com agregado sintético de argila calcinada (ASAC). Para tanto, foi utilizado um modelo viscoelástico de dano contínuo, fundamentado em trabalhos de Shapery, Kim e Lee. Os resultados com o compósito estudado (ASAC) mostraram que a perda de rigidez, baseada em uma variável interna de estado, pode ser um critério de fadiga; que o ensaio pode ser realizado para um nível de tensão; a cura de microtrincas durante os períodos de não solicitação da estrutura.
\end{abstract}

Palavras-chave: fadiga, agregados sintéticos, microtrincas.

Abstract

One of the major distresses in flexible pavings, throughout its useful life, is the fatigue cracking. This problem, in general, initiates in the base of the asphalt layer and propagates for its surface. The characterization of the asphalt mixtures, carried out in Brazil, is based on the number of cycles that cause the rupture in the fatigue test. However, such number is not a mechanical parameter, varying for different boundary conditions. The objective of the present article is to identify mechanical parameters, referring fatigue cracking, of the asphalt composite with synthetic calcinated clay aggregates (SCCA). To this end, a continuum damage viscoelastic model was used based works from Shapery, Kim and Lee. The results with the composite studied (SCCA) had shown: that the loss of rigidity, based on an internal state variable, can be a fatigue criterion; that the test can be carried through for one stress level; the healing of micro cracks during the rest periods in the structure.

Keywords: fatigue, synthetic aggregates, micro cracks.

\section{INTRODUÇÃO}

O trincamento por fadiga é um dos principais defeitos ocorrentes nos pavimentos flexíveis. Origina-se da carga repetida do tráfego e gera tensões de tração na base do revestimento. Induz, no estágio inicial, ao surgimento de microtrincas, as quais podem evoluir para trincas e, finalmente, levar à falha mecânica do mesmo. Outro importante mecanismo que incide nos pavimentos flexíveis, durante os períodos de não solicitação da estrutura, é a chamada "cura" de microtrincas, a qual pode ocasionar o aumento da vida de fadiga dos revestimentos asfálticos.

Em geral, a fadiga é tratada no dimensionamento de pavimentos flexíveis de uma forma empírico-mecanística. Particularmente, na caracterização dos compósitos asfálticos (misturas asfálticas), quanto ao trincamento por fadiga, o resultado obtido (função do número de pulsos de carga) depende das condições utilizadas no experimento. Realiza-se, comumente, o ensaio de fadiga pelo emprego de pulsos de carga harversine, com freqüência de $1 \mathrm{~Hz},(0,1 \mathrm{~s}$ de carregamento e $0,9 \mathrm{~s}$ de descanso). Define-se a vida de fadiga, na grande maioria dos trabalhos, como o número de ciclos de carga que dividem o corpo de prova ensaiado em duas partes - trata-se da denominada fratura total (critério 1) [1]. Uma outra forma utilizada (critério 2) é o número de ciclos que causam a redução no módulo resiliente (rigidez) $[2,3]$.

O desempenho mecânico de compósitos asfálticos depende de vários fatores. Pode variar o seu comportamento de elástico a viscoelástico e de plástico a viscoplástico [4]. 
O trincamento por fadiga, em regra, ocorre a temperaturas intermediárias, onde predomina o regime viscoelástico. A quantidade e o tipo de agregado utilizado na composição da mistura asfáltica, também, constituem fatores determinantes no comportamento à fadiga. Deve, ainda, ser ressaltado que os ensaios de fadiga são realizados normalmente a temperatura de $25{ }^{\circ} \mathrm{C}$, em que o compósito se comporta como um sólido viscoelástico - significa que o desempenho do mesmo dependerá, fundamentalmente, da taxa em que será solicitado. Observa-se, igualmente, quando a vida de fadiga (número de pulos de carga) é determinada segundo o critério 1 , o corpo de prova já apresenta grande perda de resistência, bem antes da fratura total ocorrer. No caso do critério 2 , a redução do módulo resiliente pode estar associada à relaxação de tensões (comportamento viscoelástico) e não à formação de trincas propriamente ditas.

Neste trabalho analisa-se o dano e a recuperação de microtrincas em misturas asfálticas do tipo CBUQ, durante o ensaio de fadiga, compostas com agregados sintéticos de argila calcinada (ASAC). Este estudo é conduzido em duas partes. Na primeira, estuda-se a caracterização viscoelástica linear do compósito, ou seja, a obtenção de uma propriedade do material que possa descrever o comportamento, sem, porém, o surgimento e evolução de microtrincas. Na segunda parte dedica-se à identificação do dano no material devido à fadiga, utilizando-se a teoria das pseudovariáveis de Schapery [5], a fim de se definir critérios mecânicos para a determinação da vida de fadiga do material.

\section{FUNDAMENTAÇÃO TEÓRICA}

\section{Caracterização viscoelástica linear}

Um material é dito viscoelástico linear quando atende ao principio da homogeneidade (equação A) e da superposição (equação B). O primeiro, dita que a reposta do material deve ser proporcional à sua solicitação, enquanto o segundo refere-se à resposta a solicitações complexas, que podem ser obtidas por meio da superposição de solicitações simples $[6,7]$. Quando um material elástico é carregado, por uma solicitação constante, sua deformação é proporcional ao carregamento e se mantém constante ao longo do tempo. No caso de materiais viscoelásticos, a deformação não se mantém constante, aumentando ao longo do tempo. Desse modo, as chaves presentes nas equações A e B indicam que a resposta é função não apenas da solicitação e sim de todo o histórico da mesma.

$$
\begin{aligned}
& \mathrm{R}\{\mathrm{AS}\}=\mathrm{AR}\{\mathrm{S}\} \\
& \mathrm{R}\left\{\mathrm{S}_{1}+\mathrm{S}_{2}\right\}=\mathrm{R}\left\{\mathrm{S}_{1}\right\}+\mathrm{R}\left\{\mathrm{S}_{2}\right\}
\end{aligned}
$$

nas quais $R, S$ e $A$ representam, respectivamente, a resposta, a solicitação e uma constante arbitrária.

Normalmente, as relações constitutivas para materiais viscoelásticos são representadas por integrais de convolução (dependência do histórico de solicitação) para condições isotérmicas, como mostram as equações C e D [5, 8, 9],

$$
\begin{aligned}
& \sigma(t)=\int_{0}^{t} E(t-\tau) \frac{\partial \varepsilon}{\partial \tau} d \tau \\
& \varepsilon(t)=\int_{0}^{t} D(t-\tau) \frac{\partial \sigma}{\partial \tau} d \tau
\end{aligned}
$$

nas quais $E(t), D(t), t$ e $\tau$ são, respectivamente, o módulo de relaxação, a função fluência (creep compliance), tempo absoluto e a variável de integração tempo entre duas mudanças de solicitação.

Existem várias formas para se determinar as funções viscoelásticas $E(t)$ e $D(t)$, seja pela realização de ensaios no domínio do tempo ou experimentos no domínio da frequiência. $\mathrm{O}$ ensaio conhecido como creep estático axial determina a função fluência, que consiste na aplicação de um carregamento instantâneo e constante ao longo do tempo com o monitoramento dos deslocamentos desenvolvidos. A função fluência pode, então, ser determinada a partir da equação $\mathrm{E}$.

$$
\mathrm{D}(\mathrm{t})=\frac{\varepsilon(\mathrm{t})}{\sigma}
$$

O ensaio de módulo de relaxação é executado para a determinação da função $E(t)$. Aplica-se um deslocamento instantâneo e constante ao longo do tempo, sendo monitorada a força necessária para manter este deslocamento. Portanto, o módulo de relaxação pode ser calculado segundo a equação $\mathrm{F}$.

$$
\mathrm{E}(\mathrm{t})=\frac{\sigma(\mathrm{t})}{\varepsilon}
$$

Os dois ensaios citados para a determinação das propriedades viscoelásticas são conceitualmente simples. No entanto, na prática, existem complicações para a execução dos mesmos, principalmente no que diz respeito a necessidade de se aplicar solicitações instantâneas. Por outro lado, o módulo complexo (propriedade viscoelástica no domínio da freqüência) pode ser obtido por meio da aplicação de pulsos de carga senoidais com o monitoramento dos deslocamentos gerados pelo pulso. Selecionou-se, para o presente trabalho, o ensaio no domínio da freqüência, uma vez que a UTM (Universal Technical Machine) pode aplicar pulsos senoidais de forma simples, o que não acontece com solicitações instantâneas. É importante ressalvar que uma vez obtida a propriedade viscoelástica no domínio da freqüência as demais propriedades no domínio do tempo podem ser calculadas por interconversão, sem a necessidade de realização de novos ensaios [6, 7].

\section{Módulo complexo}

O ensaio de módulo complexo é realizado aplicando-se pulsos de carga axial senoidais que induzem deslocamentos axiais senoidais no corpo de prova conforme as equações $G$ e H [10]. 


$$
\begin{aligned}
& \sigma(\mathrm{t})=\sigma_{0} \mathrm{e}^{\mathrm{i} w \mathrm{t}} \\
& \varepsilon(\mathrm{t})=\varepsilon_{0} \mathrm{e}^{\mathrm{i}(\mathrm{wt}-\varphi)}
\end{aligned}
$$

nas quais $\sigma_{0}$ e $\varepsilon_{0}$ são, respectivamente, as amplitudes de tensão e deformação, $\omega$ é a freqüência de aplicação de carga e o ângulo de fase $\phi$ é a diferença de tempo entre os picos de tensão e deformação.

O módulo complexo é definido, então, como o quociente entre a tensão senoidal e a deformação (equação I).

$$
\mathrm{E}^{*}=\frac{\sigma(\mathrm{t})}{\varepsilon(\mathrm{t})}=\frac{\sigma_{0}}{\varepsilon_{0}} \mathrm{e}^{\mathrm{i} \varphi}=[\cos \varphi+\mathrm{isen} \varphi]=\mathrm{E}^{\prime}+\mathrm{i} \mathrm{E}^{\prime}
$$

$E^{\prime}$ e $E$ " representam, respectivamente, a parte real e imaginaria do módulo complexo que são conhecidas como storage modulus e loss modulus. A Fig. 1 mostra esquematicamente um ensaio de módulo complexo.

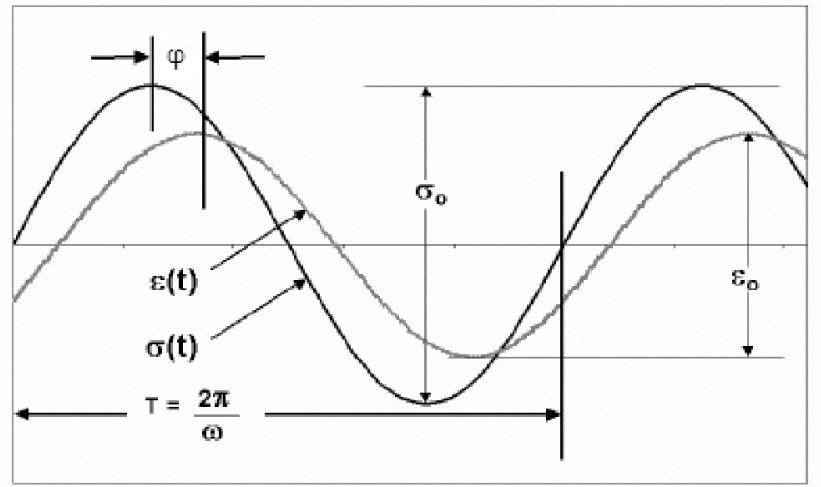

Tempo

Figura 1: Esquema de solicitação e resposta no ensaio de módulo complexo.

[Figure 1: Example of the result for the Complex Modulus test.]

O módulo dinâmico $\left|\mathrm{E}^{*}\right|$ é definido como o quociente entre a amplitude de tensão e a amplitude de deformação (equação J) e se relaciona com o storage e loss modulus pelas equações $\mathrm{K}$ e L.

$$
\begin{aligned}
& \left|E^{*}\right|=\frac{\sigma_{0}}{\varepsilon_{0}}\left|=\sqrt{\left(E^{\prime}\right)^{2}+\left(E^{\prime \prime}\right)^{2}}\right| \\
& E^{\prime}=\left|E^{*}\right| \cos \varphi \\
& E^{\prime \prime}=\left|E^{*}\right| \operatorname{sen} \varphi
\end{aligned}
$$

Vale lembrar que o módulo dinâmico e o ângulo de fase são parâmetros que dependem da freqüência de aplicação de carga. Por isso, o ensaio é realizado a várias freqüências, tendo-se valores de ângulo de fase e módulo dinâmico pontuais. Destarte, os dados pontuais obtidos necessitam serem regredidos de acordo com algum modelo mecânico, determinando-se, assim, a propriedade viscoelástica contínua.
Um dos modelos mecânicos mais simples, que se pode utilizar para a representação dos dados experimentais $E(t), D(t)$ e $\left.E^{\prime}(\omega)\right)$ té o modelo de Maxwell que consiste na associação em série de uma mola e um amortecedor, representando, respectivamente, a parte elástica e viscosa do material. Raramente este modelo representa bem o comportamento de materiais reais. No entanto, o modelo de Maxwell generalizado é capaz de representar suficientemente tais comportamentos, ficando os resultados apresentados pelo modelo praticamente indistinguíveis dos dados experimentais. O citado modelo consiste em $n$ modelos de Maxwell associados em paralelo como mostra a Fig. 2 [6-9].

Os resultados experimentais alcançados com o ensaio de módulo complexo podem ser modelados de acordo com o modelo de Maxwell generalizado, considerando-se a utilização de séries de Prony e o método da colocação [6, $7,10,11]$. As equações $\mathrm{M}$ e $\mathrm{N}$ podem ser utilizadas para modelar o comportamento observado para o storage modulus e loss modulus, respectivamente.

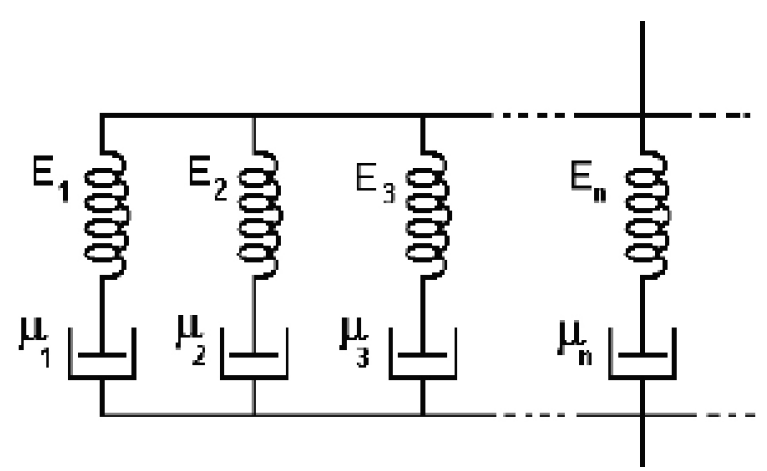

Figura 2: Modelo de Maxwell generalizado.

[Figure 2: Generalized Maxwell model.]

$$
\begin{aligned}
& E^{\prime}(\omega)=E_{0}+\sum_{i=1}^{n} \frac{\omega^{2} \rho_{i}^{2} E_{i}}{1+\omega^{2} \rho_{i}^{2}} \\
& E^{\prime \prime}(\omega)=\sum_{i=1}^{n} \frac{\omega \rho_{i} E_{i}}{1+\omega^{2} \rho_{i}^{2}}
\end{aligned}
$$

Nas equações $\mathrm{M}$ e $\mathrm{N}, p_{i}$ é o tempo de retardação, que na realidade é uma propriedade do material. A relação entre o tempo de retardação, a viscosidade e a rigidez, pode ser expressa pela equação O. É importante observar que a utilização de séries de Prony permite que os mesmos coeficientes obtidos no domínio da freqüência para as equações $\mathrm{M}$ e $\mathrm{N}$, possam ser utilizados para descrever o módulo de relaxação no domínio do tempo (equação P). Ressalta-se que o termo $E_{\infty}$ na equação $\mathrm{P}$ é igual ao termo $E_{0}$ na equação $\mathrm{M}$.

$$
\mathrm{p}_{\mathrm{i}}=\frac{\mu_{\mathrm{i}}}{\mathrm{E}_{\mathrm{i}}}
$$




$$
E(t)=E_{\infty}+\sum_{i=1}^{n} E_{i} e^{\frac{-t}{p_{i}}}
$$

\section{Dano - Materiais elásticos}

O desempenho mecânico dos materiais compósitos é afetado por mudanças microestruturais que acontecem durante o carregamento. Tais mudanças se manifestam na forma de rearranjo interno dos agregados e de aparecimento de microtrincas, entre outras. Com a aplicação do método da termodinâmica dos processos irreversíveis, foi desenvolvido uma teoria para o comportamento mecânico de materiais elásticos com mudanças microestruturais [12].

O comportamento mecânico de um material é definido em termos de uma relação entre as forças generalizadas $Q_{i}$ e os deslocamentos generalizados $q_{i}$ [12]. Estas variáveis generalizadas se transformam em variáveis físicas para diferentes situações, podendo ser forças e deslocamentos ou tensões e deformações, dependendo do problema a ser resolvido. O importante é escolhê-las de tal modo que uma seja o trabalho conjugado da outra, como mostra a equação Q [11].

$$
Q_{i} \delta \mathrm{q}_{\mathrm{i}}=\delta(\operatorname{trab})
$$

onde a repetição dos índices subentende soma sobre os mesmos e $\delta$ (trab) é o trabalho virtual.

Seguindo a teoria desenvolvida por Schapery [12], assume-se que existe uma função densidade de energia de deformação $W=W\left(q_{i}, S_{m}\right)$ de tal forma que

$$
\mathrm{Q}_{\mathrm{i}}=\frac{\partial \mathrm{W}}{\partial \mathrm{q}_{\mathrm{i}}}
$$

na qual $S_{m}$ representa as variáveis de estado internas, que pode ser, por exemplo, a mudança na geometria das trincas individuais. Para variações infinitesimais em $q_{i}$ e $S_{m}$, pode-se escrever

$$
\mathrm{dW}=\frac{\partial \mathrm{W}}{\partial \mathrm{q}_{\mathrm{i}}} \mathrm{dq}_{\mathrm{i}}+\frac{\partial \mathrm{W}}{\partial \mathrm{S}_{\mathrm{m}}} \mathrm{dS}
$$

O trabalho realizado entre um estado arbitrário 1 e um estado arbitrário 2 é dado pela integração da equação acima nesse intervalo,

$$
\mathrm{W}(2)-\mathrm{W}(1)=\left[\mathrm{W}_{\mathrm{T}}\right]_{1}^{2}+\int_{1}^{2} \frac{\partial \mathrm{W}}{\partial \mathrm{S}_{\mathrm{m}}} \mathrm{dS} \mathrm{m}_{\mathrm{m}}
$$

na qual

$$
\left[\mathrm{W}_{\mathrm{T}}\right]_{1}^{2}=\int_{1}^{2} \frac{\partial \mathrm{W}}{\partial \mathrm{q}_{\mathrm{i}}} d \mathrm{q}_{\mathrm{i}}
$$

é o trabalho realizado para deformar o material.

Define-se a função de estado $W_{S}=W_{S}\left(S_{m}\right)$ de tal forma que a equação T seja valida quando $S_{\mathrm{m}} \neq 0[11]$,

$$
-\frac{\partial \mathrm{W}}{\partial \mathrm{S}_{\mathrm{m}}}=\frac{\partial \mathrm{W}_{\mathrm{S}}}{\partial \mathrm{S}_{\mathrm{m}}}
$$

Assim sendo, pode-se escrever

$$
\int_{1}^{2} \frac{\partial \mathrm{W}}{\partial \mathrm{S}_{\mathrm{m}}} \mathrm{dS} \mathrm{m}=-\int_{1}^{2} \frac{\partial \mathrm{W}_{\mathrm{S}}}{\partial \mathrm{S}_{\mathrm{m}}} \mathrm{dS}_{\mathrm{m}}=\mathrm{W}_{\mathrm{S}}(1)-\mathrm{W}_{\mathrm{S}}(2)
$$

Tomando como referênciaW(1)= $\mathrm{W}_{\mathrm{S}}(2) \mathrm{W}_{\mathrm{T}}(1)=0$ e definido $\mathrm{W}=\mathrm{W}(2), \mathrm{W}_{\mathrm{S}}=\mathrm{W}_{\mathrm{S}}(2), \mathrm{W}_{\mathrm{T}}=\mathrm{W}_{\mathrm{T}}(2)$ a equação $\mathrm{S}$ passa a ser escrita como

$$
\mathrm{W}_{\mathrm{T}}=\mathrm{W}+\mathrm{W}_{\mathrm{S}}
$$

A equação $\mathrm{V}$ indica que o trabalho total aplicado no material $\left(W_{T}\right)$ é igual à soma da energia de deformação guardada no corpo $(W)$ com a energia dissipada durante as mudanças estruturais do corpo $\left(W_{S}\right)$ (exemplo: rearranjo interno das partículas, criação de novas bordas (trincas), etc.) [11]. Notase, também, que a equação $\mathrm{T}$ representa a lei de evolução para se obter $S_{m}=S_{m}(q)$. O primeiro membro da equação esta associado à força disponível para causar mudanças na variável interna $S_{m}$, enquanto que o segundo membro é a força necessária para provocar mudanças em $S_{m}$.

\section{Dano - Materiais viscoelásticos}

Para levar em consideração o efeito da viscoelasticidade, segue-se a teoria proposta por Schapery [5] para a qual a equação constitutiva relativa aos materiais viscoelásticos deve ser idêntica à equação constitutiva de materiais elásticos. Porém, sabe-se que as tensões e deformações não são, necessariamente, quantidades físicas no corpo viscoelástico, e sim pseudo-variáveis na forma de integrais de convolução (equações W e X) [5]. Esta substituição de variáveis físicas por pseudo-variáveis, ficou conhecida como principio da correspondência elástico-viscoelástico, pelo qual

$$
\begin{aligned}
& \varepsilon_{i j}^{R}=\frac{1}{\mathrm{E}_{\mathrm{R}}} \int_{0}^{\mathrm{t}} \mathrm{E}(\mathrm{t}-\tau) \frac{\partial \varepsilon_{\mathrm{ij}}}{\partial \tau} \mathrm{d} \tau \\
& \sigma_{i j}^{R}=\mathrm{E}_{\mathrm{R}} \int_{0}^{\mathrm{t}} \mathrm{D}(\mathrm{t}-\tau) \frac{\partial \sigma_{\mathrm{ij}}}{\partial \tau} \mathrm{d} \tau
\end{aligned}
$$

nas quais $\varepsilon_{i j}$ e $\sigma_{i j}$ são, respectivamente, a deformação e a tensão físicas, $\varepsilon_{i j}^{R}$ e $\sigma_{i j}^{R}$ são, respectivamente, a pseudodeformação e a pseudo-tensão, $E_{R}$ é uma constante arbitraria, $E(t)$ é o módulo de relaxação e $D(t)$ é a função 
fluência. Considerando-se a definição de pseudo-deformação (equação $\mathrm{W}$ ) e, a relação constitutiva uniaxial para materiais viscoelásticos (equação $\mathrm{C}$ ), pode-se escrever a equação $\mathrm{Y}$ como

$$
\sigma=\mathrm{E}_{\mathrm{R}} \varepsilon^{\mathrm{R}}
$$

Uma correspondência pode ser observada entre a equação Y e a relação constitutiva elástica. Após trabalho elaborado em 1990 [12], inúmeros autores têm utilizado essa teoria para analisar o comportamento mecânico de materiais compósitos viscoelásticos com mudanças micro estruturais (aparecimento e evolução de trincas). A utilização do princípio da correspondência elástico viscoelástico, desenvolvido, em 1984 [5], é essencial para a aplicação da teoria em materiais viscoelásticos. Alguns autores $[6,7,13-16]$ usaram a referida teoria para descrever o comportamento de misturas asfálticas, considerando-se a evolução do dano (evolução de micro trincas). Para aplicar a teoria desenvolvida [12] a materiais viscoelásticos, deve-se substituir as variáveis físicas por pseudo-variáveis. Assim, a equação R pode ser escrita como mostra a equação $Z$.

$$
Q_{i}=\frac{\partial W^{R}}{\partial q_{i}^{R}}
$$

na qual $W^{R}$ é a pseudo-energia de deformação e $\mathrm{q}^{\mathrm{R}}{ }_{\mathrm{i}}$ são os pseudo-deslocamentos generalizados. É importante observar que a lei de evolução de dano elástica (equação T) não pode ser empregada diretamente para materiais viscoelásticos, somente pelo uso das pseudo-variáveis, uma vez que as forças, disponível e necessária, para causar mudanças em $\mathrm{S}_{\mathrm{m}}$ são dependentes do tempo (histórico de solicitações) [17].

Uma lei de evolução de dano para materiais viscoelásticos similar à lei de evolução de potência para o crescimento de trincas nesses materiais (equação AA) foi empregada na elaboração de alguns trabalhos [6, 7, 13-17],

$$
\dot{\mathrm{S}} \mathrm{m}=\left(\frac{\partial \mathrm{W}^{\mathrm{R}}}{\partial \mathrm{S}_{\mathrm{m}}}\right)^{\alpha}
$$

onde $\dot{S} \mathrm{~m}$ é a taxa de evolução de dano e $\alpha$ é uma constante positiva.

Fundamentado nos resultados de ensaios dinâmicos em materiais compósitos, foi proposta a equação $\mathrm{AB}$ para a pseudo-energia de deformação no estado uniaxial [16].

$$
\begin{aligned}
& \mathrm{W}^{\mathrm{R}}=\frac{\mathrm{I}}{2} \mathrm{MC}_{1}\left(\mathrm{~S}_{1}\right)\left(\varepsilon_{\mathrm{e}}^{\mathrm{R}}\right)^{2}+\frac{\mathrm{I}}{2} \mathrm{G}\left(\frac{\varepsilon^{\mathrm{R}}}{\varepsilon_{\mathrm{L}}{ }^{\mathrm{R}}}, \varepsilon_{0}^{\mathrm{R}}\right)\left(\varepsilon_{\mathrm{e}}^{\mathrm{R}}\right)^{2} \\
& \varepsilon_{\mathrm{e}}^{\mathrm{R}}=\varepsilon^{\mathrm{R}}-\varepsilon_{\mathrm{d}}^{\mathrm{R}} \\
& \mathrm{M}=\frac{\varepsilon_{\mathrm{m}}{ }^{\mathrm{R}}}{\varepsilon_{\mathrm{m}}{ }^{\mathrm{R}}-\varepsilon_{\mathrm{d}}^{\mathrm{R}}}
\end{aligned}
$$

nas quais $I$ é a pseudo-rigidez inicial, definida como o quociente entre a pseudo-deformação máxima $\left(\varepsilon_{\mathrm{m}}{ }^{\mathrm{R}}\right)$ para o pulso inicial e sua tensão correspondente $\left(\sigma_{\mathrm{m}}\right)$ (equação AEpseudo-rigidez), $\mathrm{C}_{1}\left(\mathrm{~S}_{1}\right)$ é a função de dano e $\mathrm{G}\left(\frac{\varepsilon^{\mathrm{R}}}{\varepsilon_{\mathrm{L}}{ }^{\mathrm{R}}}, \varepsilon_{0}{ }^{\mathrm{R}}\right)$ é uma função que determina o tamanho da histerese de cada pulso. Na equação AC, $\varepsilon_{\mathrm{d}}{ }^{\mathrm{R}}$ é a pseudo-deformação causada devido à presença de micro trincas.

$$
\mathrm{S}^{\mathrm{R}}=\frac{\sigma_{\mathrm{m}}}{\mathrm{e}_{\mathrm{m}}^{\mathrm{R}}}
$$

A pseudo-energia de deformação (equação $\mathrm{AB}$ ) descreve o comportamento do material durante todos os ciclos de carregamento e descarregamento. Entretanto, neste artigo trata-se apenas com a identificação do dano. Assim, o mesmo foi determinado por pulso de carga, tendo-se como base a pseudo-deformação máxima por pulso. Considerandose a equação $\mathrm{AB}$ e fazendo-se com que $\varepsilon^{\mathrm{R}}=\varepsilon_{\mathrm{m}}{ }^{\mathrm{R}}$, a equação AF pode ser escrita na forma

$$
\begin{aligned}
& \mathrm{W}^{\mathrm{R}}=\frac{1}{2} \quad \mathrm{C}_{1}\left(\mathrm{~S}_{1}\right) \varepsilon_{\mathrm{m}}{ }^{\mathrm{R}} \varepsilon_{\mathrm{me}}{ }^{\mathrm{R}} \\
& \varepsilon_{\mathrm{me}}{ }^{\mathrm{R}}=\varepsilon_{\mathrm{m}}{ }^{\mathrm{R}}-\varepsilon_{\mathrm{d}}{ }^{\mathrm{R}}
\end{aligned}
$$

Da equação R pode-se, então, escrever:

$$
\sigma_{\mathrm{m}}=\mathrm{IC}_{1}\left(\mathrm{~S}_{1}\right) \varepsilon_{\mathrm{m}}{ }^{\mathrm{R}}
$$

Note-se que a função de dano $C_{1}\left(S_{1}\right)$ na equação $A H$ é idêntica a pseudo-rigidez, validando-a, assim, como um bom parâmetro para a identificação do dano por fadiga no material.

\section{MATERIAIS E MÉTODOS}

\section{Agregados, ligante e filer}

Selecionou-se o solo (amostra PUC) para a confecção dos agregados sintéticos de argila calcinada (ASAC) [18]. Foi caracterizado segundo procedimentos geotécnicos típicos: análise granulométrica [19] e determinação dos limites de consistência [20,21]. O processo de confecção dos ASACs consistiu em: homogeneização da amostra com adição de água; moldagem de massas de solo em formato cilíndrico, para posterior corte por meio de telas, com abertura da malha conforme dimensão desejada para os agregados; secagem dos agregados à sombra, acondicionamento dos agregados em jarros cerâmicos e calcinação, em forno elétrico, à temperatura aproximada de $900{ }^{\circ} \mathrm{C}$. Após a obtenção dos agregados calcinados, foram realizados os testes de qualificação, estabelecidos pelo Departamento Nacional de Estradas de Rodagens (DNER), atual Departamento Nacional de Infra-Estrutura 
de Transportes (DNIT): a) Seleção Expedita pelo Processo de Fervura - Ensaio de Autoclave [22], b) Perda de Massa após Fervura [23] e, c) Desgaste por Abrasão "Los Angeles" [24]. Além do agregado sintético, duas areias, uma proveniente do município de Coari - AM (Areia 1) e a outra procedente da região de Urucu - AM (Areia 2), foram utilizadas na composição da mistura asfáltica. Com estes materiais foram realizadas análises granulométricas [25], e a determinação do Loose Unit Weight (Wul) e do Rodded Unit Weight (Wur) [26]. Para os agregados calcinados, também, foram determinados: Apparent Specific Gravity (Gsa), Bulk Specific Gravity (Gsb), Bulk Specific Gravity in the condition Saturated Surface Dry (Gsb ssd) e Absorption [27]. Os parâmetros Gsb, Gsb ssd e Absorption, igualmente, foram encontrados para as areias [28]. Classificou-se o Cimento Asfáltico de Petróleo - CAP 50/70, segundo critério Superpave $[29,30]$ e, obteve-se a massa específica real [31] e a granulometria do cimento Portland (fíler) [32].

\section{Dosagem do compósito}

A composição da mistura granulométrica (ASAC, Areia 1, Areia 2 e cimento Portland) seguiu a Faixa Superpave, com Diâmetro Máximo Nominal (DMN) de 12,5 mm [29, 30]. As porcentagens relativas a cada um dos agregados componentes da mistura, incluindo o fíler, foram definidas de acordo com o método de Bailey [33].

$\mathrm{O}$ teor de projeto foi determinado com base nos valores de Volume de Vazios (Vv) e na Relação Betume - Vazios (RBV), segundo os procedimentos descritos pelo $3^{\circ}$ Distrito Rodoviário Federal - $3^{\circ}$ DRF [34].

O método Marshall [35] foi adotado na compactação dos corpos de prova, visando à definição da dosagem a ser utilizada na realização dos ensaios. Objetivando-se simular o efeito de envelhecimento de curto prazo, as misturas ficaram, antes da compactação, $2 \mathrm{~h}$ em estufa a $144{ }^{\circ} \mathrm{C}$ [36]. Definiram-se as temperaturas de preparo e compactação [37], que resultaram em $158{ }^{\circ} \mathrm{C}$ a $167{ }^{\circ} \mathrm{C}$ e $148{ }^{\circ} \mathrm{C}$ a $157{ }^{\circ} \mathrm{C}$, respectivamente. Utilizou-se o Rice Test [38] para o cálculo da Densidade Específica Máxima da mistura (Gmm), segundo um teor de ligante de $6,0 \%$, permitindo-se, assim, a determinação da fração de ligante absorvida pelos agregados (Pba). Depois de encontrada a $\mathrm{Gmm}$, para o teor inicial de ligante, os valores concernentes aos outros teores, foram calculados pela Superpave $[29,30]$.

\section{Programa experimental}

O ensaio para a obtenção do módulo dinâmico foi realizado à tração axial, similar ao ensaio de fadiga, e a $25^{\circ} \mathrm{C}$. As frequiências de aplicação de carga foram $0,01,0,05,0,1$, $0,5,1,5,10$ e $25 \mathrm{~Hz}$.

$\mathrm{O}$ ensaio de fadiga foi realizado em quatro estágios de carga a $25^{\circ} \mathrm{C}$ e na freqüência de $1 \mathrm{~Hz}$. Inicialmente, foram aplicados um total de 100 pulsos com carga de $0,5 \mathrm{kN}$, que se presume ter gerado pouco dano ao corpo de prova, seguido de $50 \mathrm{~s}$ de descanso. Após este repouso, 300 pulsos foram aplicados com carga de $0,75 \mathrm{kN}$, seguidos de 200 $\mathrm{s}$ de descanso. Em seguida, foram aplicados 50 pulsos de carga de $1,0 \mathrm{kN}$, com $200 \mathrm{~s}$ de repouso, como o objetivo de analisar algum tipo de recuperação na função de dano (pseudo-rigidez), indicando, deste modo, algum tipo de recuperação de micro trincas. Por fim, o corpo de prova foi submetido a pulsos com carga $1 \mathrm{kN}$, até a sua ruptura total.

No ensaio axial à tração, moldaram-se os corpos de prova com dimensões finais de $100 \mathrm{~mm}$ de diâmetro e $200 \mathrm{~mm}$ de altura. Executou-se a compactação de forma a se obter a mesma densidade aparente (Gsb), alcançada na dosagem da mistura para o teor de projeto. Procurando-se minimizar o efeito de borda as medidas de deslocamento no corpo de prova foram realizadas com micros LVDTs acoplados a uma distância de $150 \mathrm{~mm}$, a $25 \mathrm{~mm}$ de cada face.

\section{RESULTADOS E DISCUSSÃO}

\section{Caracterização das amostras e da mistura}

As Tabelas I e II apresentam os resultados da determinação da textura e dos limites de consistência da amostra PUC. De acordo com a classificação da American Association of State Highway and Transportation Officials/ Transportation Research Board (AASHTO/TRB), o solo PUC foi classificado como A7-5 e, pelo Sistema Unificado de Classificação dos Solos (SUCS), como CH. Na Tabela III são mostrados os resultados dos ensaios de qualificação, aos quais foram submetidos os agregados sintéticos, onde indicam que atenderam às especificações.

Classificou-se o Cimento Asfáltico de Petróleo - CAP 50/70 conforme critério Superpave, como PG 64-22. O cimento Portland apresentou massa específica real 3,150 $\mathrm{g} / \mathrm{cm}^{3}$, e granulometria satisfazendo ao padronizado para material de enchimento, conforme estabelece a norma DNER ME 367/97. A Tabela IV indica os resultados com os ensaios de caracterização dos agregados componentes da mistura asfáltica.

Tabela I - Composição textural da Amostra PUC. [Table I - Textural composition of the PUC sample.]

\begin{tabular}{cc}
\hline Textura & Resultado \\
\hline Areia & $15 \%$ \\
Silte & $41 \%$ \\
Argila & $44 \%$ \\
passando na \# $0,075 \mathrm{~mm}$ & $92,0 \%$ \\
\hline
\end{tabular}

Tabela II - Índices de Consistência da amostra PUC. [Table II - Consistency limits of the PUC sample.]

\begin{tabular}{cc}
\hline Índices & Resultado \\
\hline Limite de Liquidez - LL & $55 \%$ \\
Limite de Plasticidade - LP & $31 \%$ \\
Índice de Plasticidade - IP & $24 \%$ \\
\hline
\end{tabular}


Tabela III - Ensaios de qualificação do ASAC. [Table III - Qualification tests - SCCA.]

\begin{tabular}{ccc}
\hline Ensaio & $\begin{array}{c}\text { Valores } \\
\text { Limites }\end{array}$ & $\begin{array}{c}\text { Amostra } \\
\text { PUC }\end{array}$ \\
\hline Variação de textura e forma & Não & Não \\
DNER ME 223/94 & Varia & Varia \\
Perda de massa após fervura & Máx & $0,14 \%$ \\
DNER ME 225/94 & $10 \%$ & \\
Abrasão "Los Angeles" & Máx & $43 \%$ \\
DNER ME 222/94 & $45 \%$ & \\
\hline
\end{tabular}

Tabela IV - Ensaios de caracterização dos agregados. [Table IV - Aggregates characterization tests.]

\begin{tabular}{cccc}
\hline Amostra & ASAC PUC & Areia 1 & Areia 2 \\
\hline Gsb $\left(\mathrm{g} / \mathrm{cm}^{3}\right)$ & 1,730 & 2,604 & 2,601 \\
Gsb ssd $\left(\mathrm{g} / \mathrm{cm}^{3}\right)$ & 2,076 & 2,677 & 2,674 \\
Gsa $\left(\mathrm{g} / \mathrm{cm}^{3}\right)$ & 2,644 & - & - \\
Absorption $(\%)$ & 20,00 & 0,74 & 0,73 \\
Wul $\left(\mathrm{kg} / \mathrm{m}^{3}\right)$ & 1021,25 & - & - \\
Wur $\left(\mathrm{kg} / \mathrm{m}^{3}\right)$ & 1107,31 & 1710,82 & 1583,30 \\
\hline
\end{tabular}

Como já era de se esperar, o material sintético (Amostra PUC), por se tratar de agregado leve, apresentou sua densidade (Específica Aparente, Solta e Compactada) inferior aos respectivos valores correspondentes às Areias (1 e 2). Os ASACs apresentaram, também, um alto potencial de absorção (20\%), podendo indicar que a mistura composta por esse tipo de material, provavelmente, necessitará de uma maior quantidade de ligante, em relação às misturas com agregados naturais [39].

A Fig. 3 apresenta o enquadramento da curva granulométrica na Faixa Superpave, com DMN de 12,5 mm, composta por: ASAC $(60,05 \%)$, Areia $1(19,68 \%)$, Areia 2 $(16,85 \%)$ e cimento Portland $(3,42 \%)$.
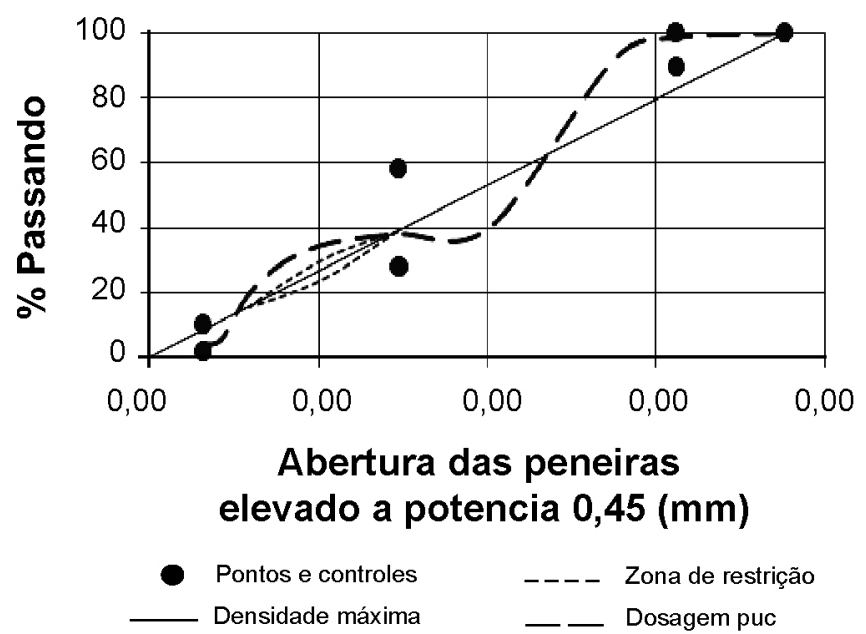

Figura 3: Curva granulométrica da mistura avaliada.

[Figure 3: Grain size of the evaluated mixture.]
Observa-se na Fig. 4 os valores obtidos para T1, T2, T3 e $\mathrm{T} 4$, ou seja, $6,57 \%, 5,79 \%, 6,35 \%$ e $6,79 \%$, respectivamente. Pela média aritmética dos dois valores centrais (T1 e T3), definiu-se o teor de projeto " $T$ ", que resultou no valor de $6,46 \%$. A absorção de ligante, pelo agregado sintético, apresentou o valor de $0,70 \%$. O resultado da $\mathrm{Gmm}$ para teor de projeto da mistura $(6,46 \%)$ foi de $1,912 \mathrm{~g} / \mathrm{cm}^{3}$.

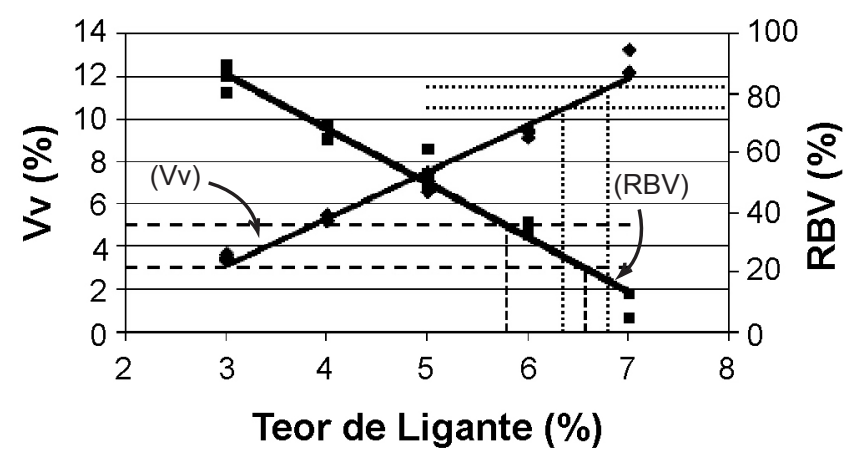

- $V_{V} \diamond V_{V}$ - Linear (Vv) - Linear (RBV)

Figura 4: Determinação do teor de ligante pelo $3^{\circ} \mathrm{DRF}$.

[Figure 4: Determination of asphalt optimum content according $3^{\circ} \mathrm{DRF}$.]

\section{Caracterização linear}

Nos deslocamentos medidos pelos LVDTs (principalmente a baixos deslocamentos) é inevitável certo nível de ruído elétrico, sendo necessário suavizar as leituras. Dois métodos podem ser empregados: no primeiro utiliza-se uma função de suavização (distribuição normal de Gauss) e, no segundo, faz-se à regressão dos deslocamentos obtidos, como no presente trabalho. A Fig. 5 mostra os deslocamentos medidos e regredidos para a freqüência de $0,5 \mathrm{~Hz}$.

Após a regressão dos dados constantes na Fig. 5 e utilizando-se a equação AI, o módulo dinâmico foi calculado com a equação $\mathrm{J}$ e o ângulo de fase foi computado conforme

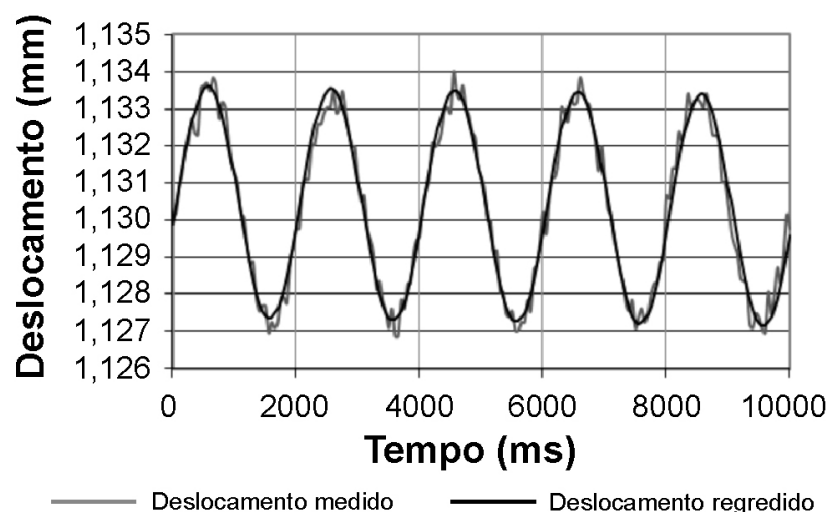

Figura 5: Deslocamento medido e deslocamento regredido. [Figure 5: Measured displacement and fitted displacement.] 
mostra a Fig. 1, considerando-se a freqüência. As Figs. 6 e 7 apresentam, respectivamente, os resultados de módulo dinâmico e ângulo de fase para as diferentes freqüências de ensaio.

$$
f(t)=a+b t+\operatorname{csen}(\omega t+\varphi)
$$

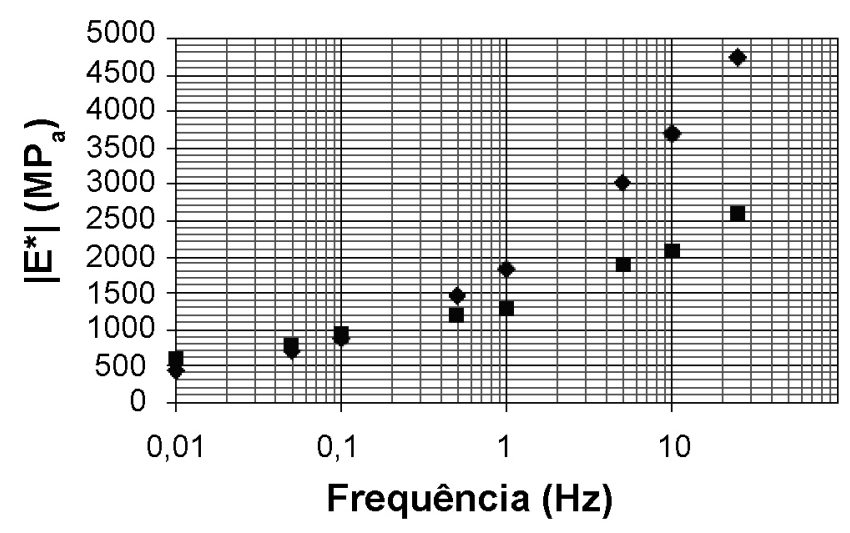

- $\left|E^{*}\right| A S A C$

$\left|E^{\star}\right|$ Calcario

Figura 6: Módulo dinâmico para a mistura com ASAC e para uma mistura com calcário.

[Figure 6: Dynamic Module for the mixture with SCCA and for the a mixture using limestone.]

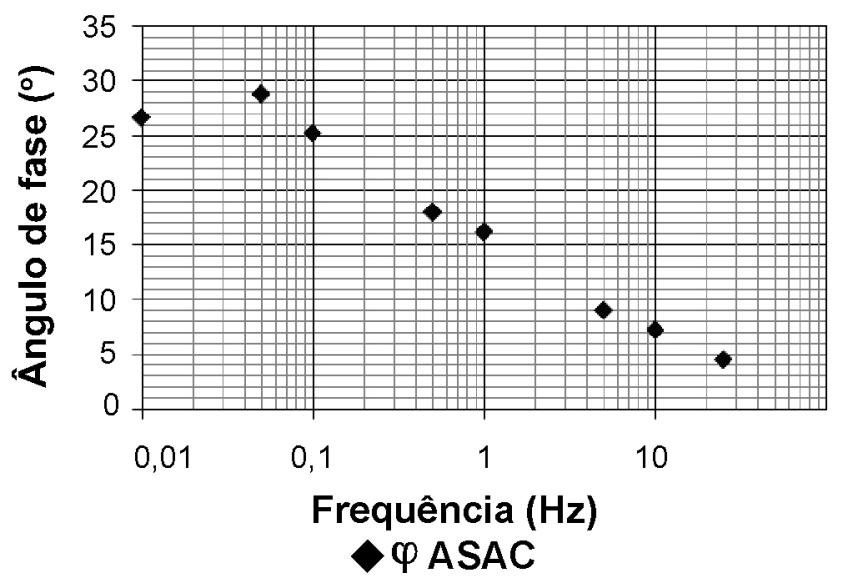

Figura 7: Ângulo de fase para mistura com ASAC.

[Figure 7: Phase Angle for the mixture with SCCA.]

Na Fig. 6 tem-se o módulo dinâmico para dois compósitos asfálticos, um com ASAC e o outro com um agregado convencional (calcário), ensaiado à compressão axial [10], observando-se que o valor do módulo dinâmico depende da freqüência de aplicação de carga. Nota-se que, em baixas freqüências, ambas as misturas possuem valores de $\left|E^{*}\right|$ equivalentes. Para altas freqüências, há uma maior diferença entre os resultados para a mistura com ASAC e a mistura com calcário.

A Fig. 7 mostra o ângulo de fase para cada frequiência de ensaio. Como já era de se esperar, o mesmo diminui com o aumento da frequiência. Com os dados apresentados nas Figs. 6 e 7 pode-se calcular o valor de E' $(\omega)$ (storage modulus) com a equação K. Computados os valores de E', utilizou-se o método da colocação para determinar os valores dos parâmetros para as equações M e P. A obtenção destes parâmetros (equação $\mathrm{P}$ ) é de vital importância para a análise do comportamento do compósito quanto à fadiga.

A Fig. 8 condensa os valores experimentais de $E^{\prime}(\omega)$ (valores pontuais), e os valores de $E^{\prime}(\omega)$ e $E^{\prime}(t)$, computados a partir das equações $\mathrm{M}$ e P. Os parâmetros utilizados para determinar os dados estão apresentados na Tabela V.

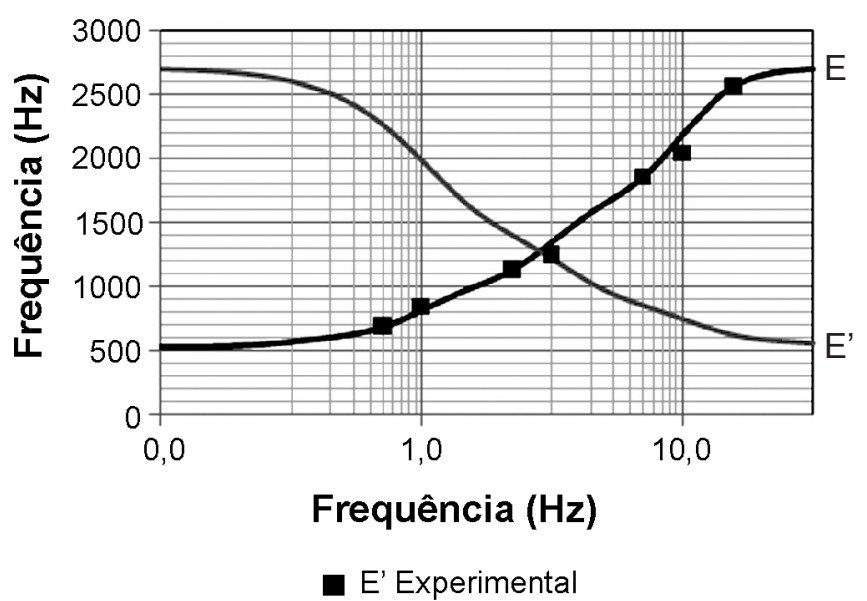

Figura 8: Resultados para o storage modulus e para o módulo de relaxação.

[Figure 8: Results for the storage modulus and for the relaxation modulus.]

Tabela V - Parâmetros obtidos por regressão para as equações $\mathrm{M}$ e P.

[Table $V$ - Parameters obtained by regression for the $M$ and $P$ equations.]

\begin{tabular}{cc}
\hline$E_{0}$ ou $E_{\infty}(\mathrm{MPa})$ & 529,122 \\
\hline$E_{i^{\prime}}(\mathrm{MPa})$ & $\rho_{i^{\prime}}(\mathrm{s})$ \\
1037,527 & 0,1 \\
661,3273 & 1 \\
409,705 & 10 \\
73,48403 & 100 \\
\hline
\end{tabular}

Observa-se, na Fig. 8, que a equação M, juntamente com os dados da Tabela V, proporciona um bom enquadramento dos dados experimentais, validando, por conseguinte, os parâmetros constantes na referida tabela. É importante ressaltar que a evolução das funções $E^{\prime}(\omega)$ e E' $(t)$ apresentam uma forma em "S", com um limite mínimo e um limite máximo. Tais limites, vistos na Fig. 8, são impostos pela regressão dos dados experimentais. Outros limites máximos e mínimos poderiam ser determinados no caso de um número de dados experimentais maior, considerando-se o princípio da superposição tempo-temperatura, onde o experimento (módulo dinâmico) é realizado a várias temperaturas e, podendo-se, posteriormente, montar uma curva mestra. 
Em outro trabalho, realizado pelo Grupo de Geotecnia, foi detalhado este tipo de ensaio a várias temperaturas e a montagem da curva mestra [40].

\section{Dano induzido por fadiga}

A Fig. 9 indica os resultados para as três forças utilizadas na seqüência de ensaios e em determinados pulsos, para o compósito com ASAC. Os gráficos apresentados à esquerda $\left(a_{1}, b_{1}, c_{1}\right.$ e d $\left.d_{1}\right)$ estão em função da deformação axial e, à

$\begin{array}{lllll}-0,00008 & -0,00006 & -0,00004 & -0,00002 & -0,00000\end{array}$

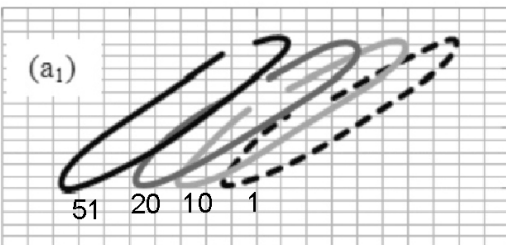

$-0,00$

$-20,00$

$-40,00$

$-60,00$

$-80,00$

$1-10$

20

$-51$

$\begin{array}{lllll}-0,00020 & -0,00015 & -0,00010 & -0,00005 & 0,00000\end{array}$

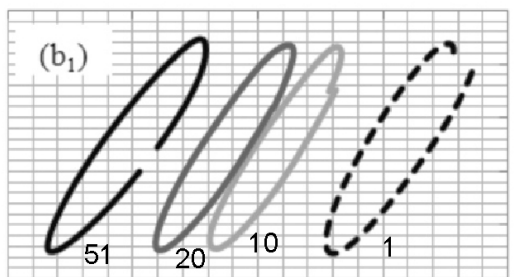

$-0,00$

$-20,00$

$-40,00$

$-60,00$

$-80,00$

$-100,00$

-..-.- $1-10$

$-20$

$-51$
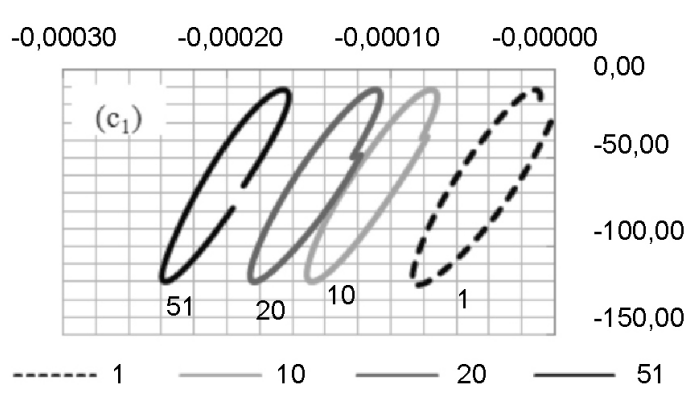

$\begin{array}{llll}-0,0025 & -0,0015 & -0,0005 & 0,0005 \\ 0,00\end{array}$

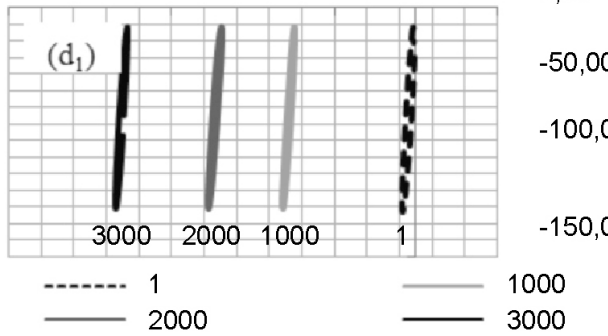

direita $\left(a_{2}, b_{2}, c_{2}\right.$ e $\left.d_{2}\right)$, estão segundo a pseudo-deformação axial. As pseudo-deformações foram calculadas a partir da equação W, empregando-se o módulo de relaxação (ensaio de módulo dinâmico). A solução da equação $\mathrm{W}$ foi obtida a partir de integração numérica considerando-se a taxa de deformação linear entre sucessivos passos, referentes a tempos consecutivos.

Observa-se, na Fig. 9, que as curvas tensão $\times$ deformações movem-se para a esquerda com o aumento do número de pulsos de carga e, também, que a distância entre os pulsos
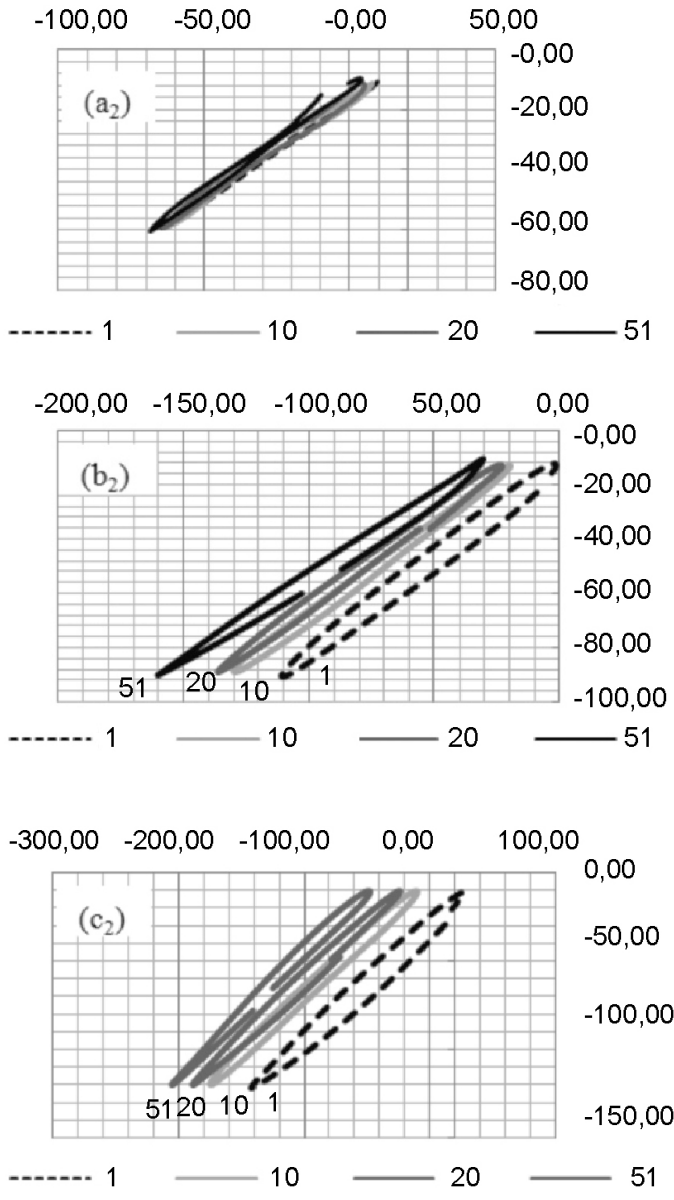

$\begin{array}{lllllllll}-1200 & -1000 & -800 & -600 & -400 & -200 & 0 & 200 & 0,00\end{array}$

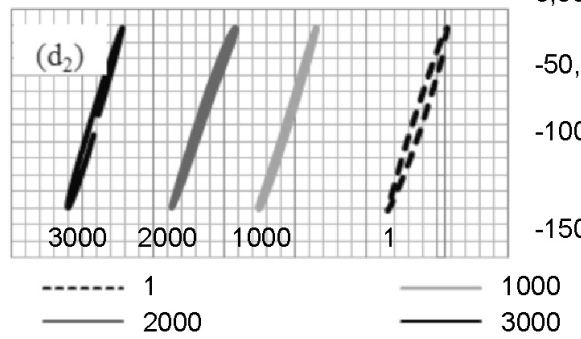

Figura 9: Resultados obtidos no ensaio de fadiga, a d ) deformação axial 0,50 kN, $\mathrm{a}_{2}$ ) pseudo-deformação axial 0,5 kN, $\mathrm{b}_{1}$ ) deformação axial $0,75 \mathrm{kN}, \mathrm{b}_{2}$ ) pseudo-deformação axial $0,75 \mathrm{kN}, \mathrm{c}_{1}$ ) deformação axial 1,0 kN, $\mathrm{c}_{2}$ ) pseudo-deformação axial 1,0 kN, $\mathrm{d}_{1}$ ) deformação axial 1,0 $\mathrm{kN}, \mathrm{d}_{2}$ ) pseudo-deformação axial 1,0 kN.

[Figure 9: Results obtained for the fatigue test, a1) axial strain $0.50 \mathrm{kN}$, a2) axial pseudo-strain $0.5 \mathrm{kN}, \mathrm{b1}$ ) axial strain $0.75 \mathrm{kN}$, b2) axial pseudo-strain $0.75 \mathrm{kN}, \mathrm{cl}$ ) axial strain $1.0 \mathrm{kN}, \mathrm{c} 2$ ) axial pseudo-strain $1.0 \mathrm{kN}$, d1) axial strain $1.0 \mathrm{kN}$, d2) axial pseudo-strain $1.0 \mathrm{kN}$.] 
está diretamente ligada à amplitude da carga aplicada. Esse comportamento pode ser devido à fluência viscoelástica (creep) ou ao desenvolvimento de microtrincas no compósito (dano).

Aplicando-se a equação $\mathrm{W}$ às curvas tensão $\times$ deformação da Fig. 9, pode-se eliminar dos resultados a fluência viscoelástica. Deste modo, qualquer deslocamento dos pulsos para a esquerda será devido ao surgimento de microtrincas $\left(\varepsilon_{\mathrm{d}}^{\mathrm{R}}\right)$ (similar ao comportamento elástico). Tem-se, ainda, nessa figura, que na curva tensão $\times$ pseudodeformação (Fig. 12a), os pulsos de carga de $0,5 \mathrm{kN}$ não induzem danos significativos na amostra. No caso das curvas tensão $\times$ pseudo-deformação (Figs. $9 b_{2}$ e $9 c_{2}$ ), observa-se um aumento na $\varepsilon_{\mathrm{d}}{ }^{\mathrm{R}}$, levando-se, assim, a uma redução da pseudo-rigidez da amostra, indicando o desenvolvimento de microtrincas. Na Fig. $9 d_{2}$ percebe-se a evolução da $\varepsilon_{d}{ }^{R}$ de forma evidente, pois foram plotados pulsos de carga isolados e espaçados de 1000 ciclos (pulsos).

Na Fig. 10 tem-se a curva tensão $\times$ pseudo-deformação, com carga de 1,0 kN para o último pulso, antes do período de $200 \mathrm{~s}$ de descanso e, para o primeiro pulso de carga após o período de descanso.

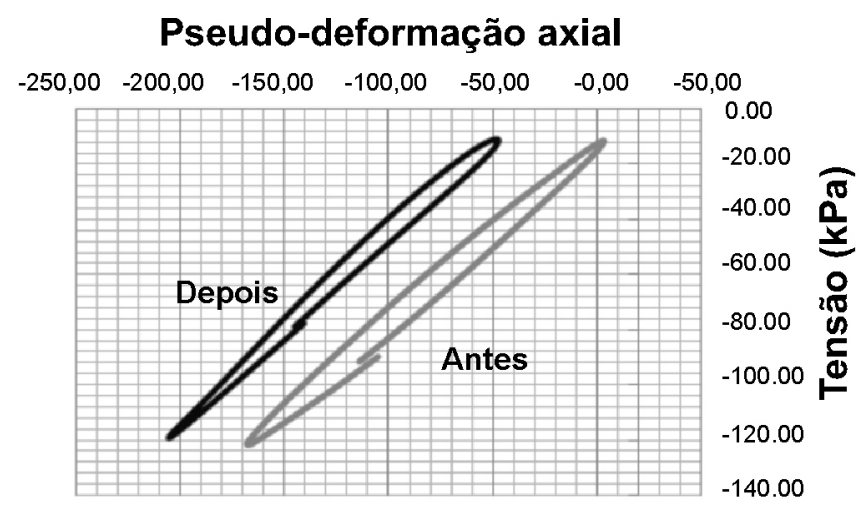

Figura 10: Tensão $\times$ Pseudo-deformação antes e após o período de descanso.

[Figure 10: Stress $\times$ pseudo-strain before and after the rest period.]

Tem-se, na Fig. 10, que em seguida ao período de descanso, ocorreu um aumento na pseudo-rigidez, devido a uma recuperação da $\varepsilon_{\mathrm{d}}{ }^{\mathrm{R}}$, sugerindo certo nível de cura das microtrincas. Para os três níveis de carga pode-se calcular o dano na amostra $C_{1}\left(S_{1}\right)$ por meio da equação $A H$. A variável interna de estado $\left(\mathrm{S}_{1}\right)$ é obtida segundo a equação $\mathrm{AA}$, para um valor de $\alpha$ igual a 1.592. O valor de $\alpha$, à tensão controlada, foi calculado para $\alpha=1 / \mathrm{m}$, onde $\mathrm{m}$ é a inclinação da parte linear da curva de creep $[13,16]$.

A Fig. 11 mostra a evolução do dano para os níveis de carga ensaiados. Nota-se, para a carga de $0,5 \mathrm{kN}$, que a evolução do dano é insignificante nos 100 pulsos de aplicação de carga. No entanto, para os 300 pulsos de aplicação de carga, com amplitude de $0,75 \mathrm{kN}$, observa-se uma evolução significativa do dano. Para os 4390 pulsos de $1 \mathrm{kN}$, temse a evolução do dano até a ruptura. A descontinuidade
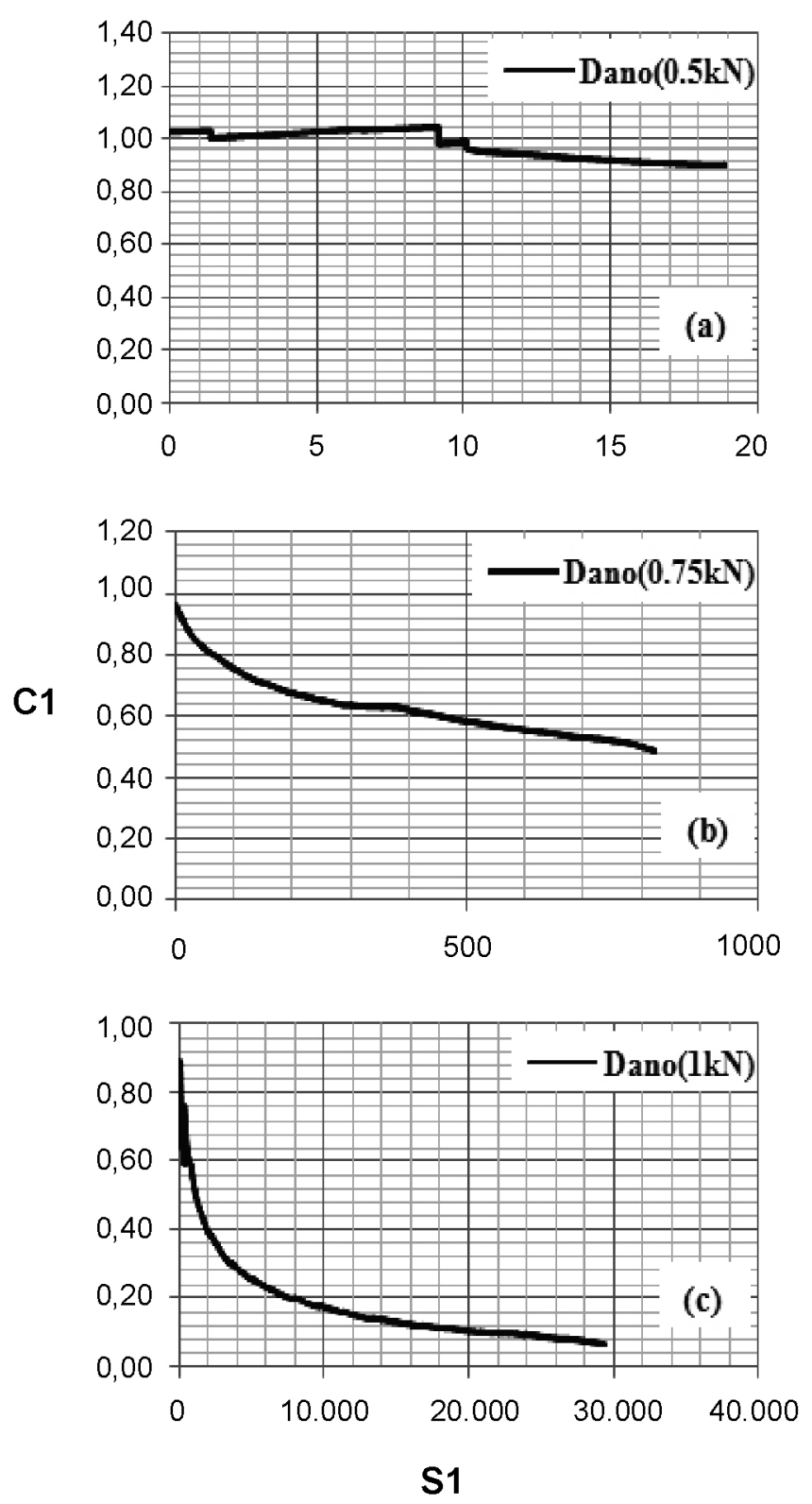

Figura 11: Evolução do dano para as amplitudes de carregamento.a) 0,5 kN 50 pulsos, b) 0,75 kN 300 pulsos, c) 1,0 kN 4390 pulsos.

[Figure 11: Damage evolution for the loading amplitudes, a) 0.5 kN 50 pulses, b) $0.75 \mathrm{kN} 300$ pulses, c) $1.0 \mathrm{kN} 4390$ pulses.]

observada na Fig. 11c é devido à cura de microtrincas (Fig. 10), podendo, além disso, ser visualizada de forma mais consistente na Fig. 12.

A Fig. 11 pode ser utilizada como critério de parada nos ensaios de trincamento por fadiga, determinandose, por exemplo, que a fadiga do material ocorre quando o parâmetro de dano $\mathrm{C}_{1}\left(\mathrm{~S}_{1}\right)$ atingir um determinado nível em relação ao inicial (20\% do valor inicial). Percebe-se, na Fig. $11 \mathrm{c}$, que o valor de $20 \%$ de $C_{l}\left(S_{l}\right)$ é alcançado com aproximadamente 2000 pulsos de carga e a fratura total da amostra ocorre somente com 4390 pulsos. A Fig. 12 evidencia a descontinuidade apresentada na Fig. 11c, devido à cura de microtrincas (período de descanso), juntamente com a curva de dano indicada na Fig. 11b. 


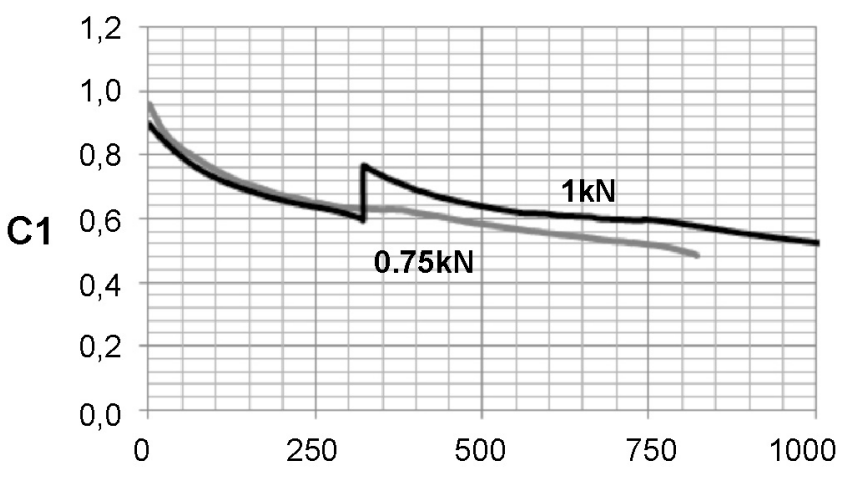

Figura 12: Descontinuidade no dano devido ao período de descanso.

[Figure 12: Discontinuity in the damage due to the rest period.]

A Fig. 12 mostra que as curvas de dano não são significativamente distintas para diferentes amplitudes de carga, tornando esse parâmetro, como já era esperado, independente do nível de tensão. Comparando-se as duas curvas, tem-se uma redução no dano devido ao período de descanso (descontinuidade). Após este período, com o aumento do número de ciclos, percebe-se um aumento drástico no dano do material, restabelecendo-se, assim, o estado de dano anterior ao período de descanso (mesma inclinação da curva sem período de descanso). Evidentemente, o dano aumenta até encontrar a curva do material sem este período. Na Fig. 12, fica evidente que o número de ciclos necessário para atingir certo nível de dano aumenta com o período de descanso.

\section{CONCLUSÕES}

Foi abordada a identificação do dano induzido por fadiga, em compósitos viscoelásticos - misturas asfálticas com ASAC, segundo o princípio da correspondência elásticaviscoelástica (pseudo-variáveis), visando eliminar o efeito viscoso nestes compósitos (creep e relaxação). A análise dos resultados, referentes ao ensaio de fadiga, segundo essa ótica mecanística, mostrou: a) a perda de rigidez devido à formação de trincas, possibilitando a utilização de um critério de fadiga, baseado em uma variável interna de estado, que representa o dano no material; b) a redução do tempo no ensaio de fadiga, uma vez que, este é realizado, em geral, em três níveis de tensão, enquanto os resultados alcançados neste estudo indicaram que o parâmetro de dano independe do nível de tensão, sendo necessário, apenas, um nível de tensão para determiná-lo; c) a identificação da cura de microtrincas (recuperação da rigidez) nos períodos de descanso.

\section{REFERÊNCIAS}

[1] L. M. G. Motta, J. Medina, A. M. Sousa, Caracterização de módulos de resiliência e de fadiga de misturas asfálticas brasileiras, in $16^{\circ}$ Encontro de Asfalto do IBP, 2002, Rio de Janeiro, RJ. Anais do $16^{\circ}$ Encontro de Asfaltov, cd (2002). [2] M. G. R. Santos, Estudo do comportamento de agregados sintéticos de argila calcinada para uso em revestimentos asfálticos para Manaus, Diss. Mestrado, Universidade de Brasília - UnB, Programa de Mestrado em Geotecnia, Brasília, DF (2007).

[3] C. A. Frota, C. L. Silva, D. M. Melo, F. R. G. Nunes, Novos materiais para a construção de pavimentos rodoviários, Relatório de Pesquisa, Universidade Federal do Amazonas - UFAM, Grupo de Geotecnia - GEOTEC, Manaus, AM (2006).

[4] A. Sides, J. Uzan, M. Perl, A comprehensive viscoelastoplastic characterization of sand-asphalt under compression and tension cyclic loading, ASTM, J. Testing Evaluation 13 (1985) 59.

[5] R. A. Schapery, Correspondence principles and a generalized J-integral for large deformation and fracture analysis of viscoelastic media, Int. J. Fracture 25 (1984) 195.

[6] G. Chehab, Characterization of asphalt concrete in tension using a viscoelastoplastic model, Ph.D. Diss., North Carolina State University, Raleigh, NC, EUA (2002).

[7] N. H. Gibson, A Viscoelastoplastic continuum damage model for compressive behavior of asphalt concrete, Ph.D. Diss., North Carolina State University, Raleigh, NC, EUA (2006).

[8] F. V. Souza, J. B. Soares, Previsão do comportamento mecânico de misturas asfálticas a partir dos resultados do ensaio de creep estático, Anais do XVI Cong. Pesquisa e Ensino em Transportes, ANPET, Natal, RN (2002) 29.

[9] F. V. Souza, J. B. Soares, Efeito da consideração do comportamento viscoelástico linear do revestimento no cálculo de tensões e deslocamentos em pavimentos asfálticos, Anais do XVII Cong. Pesquisa e Ensino em Transportes, ANPET, Rio de Janeiro, RJ (2003).

[10] M. H. King, Determination of dynamic moduli in uniaxial compression for North Caroline hot mix asphalt concrete, M.Sc. Diss., North Carolina State University, Raleigh, NC, EUA (2004).

[11] S. W. Park, R. A. Schapery, methods of interconversion between linear viscoelastic material functions. Part I - A numerical method based on prony series, Int. J. Solids Structures 36 (1999) 1653.

[12] R. A. Schapery, A theory of mechanical behavior of elastic media with growing damage and other changes in structure, J. Mechanics Phys. Solids 38 (1990) 215.

[13] H. J. Lee, Y. R. Kim, Viscoelastic continuum damage model of asphalt concrete with healing, J. Eng. Mechanics 124 (1998) 215.

[14] H. J. Lee, Uniaxial constitutive modeling of asphalt concrete using viscoelasticity and continuum damage theory, Ph.D. Diss., North Carolina State University, Raleigh, NC, EUA (1996).

[15] S. W. Park, Y. R. Kim, R. A. Schapery, A viscoelastic continuum camage model and its application to uniaxial behavior of asphalt concrete, Mechanics Mater. 24, 4 (1996) 241.

[16] H. J. Lee, Y. R. Kim, Viscoelastic constitutive model for asphalt concrete under cyclic loading, J. Eng. Mechanics 124, 1 (1997) 32. 
[17] S. W. Park, R. A. Schapery, A thermoviscoelastic constitutive model for particulate composites with growing damage, JANNAF Structures \& Mechanical Behavior (1994).

[18] C. A. Frota, C. L. Silva, M. G. R. Santos, R. V. Silva, Caracterização de solos para obtenção de agregados sintéticos de argila calcinada, $35^{\text {a }}$ Reunião Anual de Pavimentação, Rio de Janeiro, RJ (2004).

[19] ABNT, NBR 7181, Solo - Análise granulométrica, Associação Brasileira de Normas Técnicas, Rio de Janeiro, RJ (1984).

[20] ABNT, NBR 6459, Solo - Determinação do limite de liquidez, Associação Brasileira de Normas Técnicas, Rio de Janeiro, RJ (1984).

[21] ABNT, NBR 7180, Solo - Determinação do limite de plasticidade, Associação Brasileira de Normas Técnicas, Rio de Janeiro (1984).

[22] DNER, ME 223/94 - Argilas para a fabricação de agregados sintéticos de argila calcinada: Seleção expedita pelo Processo de Fervura, Departamento Nacional de InfraEstrutura de Transportes, Rio de Janeiro, RJ (1994).

[23] DNER, ME 225/94 - Agregado sintético de argila calcinada: Determinação da perda de massa após fervura, Departamento Nacional de Infra-Estrutura de Transportes, Rio de Janeiro, RJ (1994).

[24] DNER, ME 222/94 - Agregado sintético fabricado com argila: Desgaste por abrasão, Departamento Nacional de Infra-Estrutura de Transportes, Rio de Janeiro, RJ (1994).

[25] ASTM, C 136-95 - Standard test method for sieve analysis of fine and coarse aggregates, American Association of State Highway and Transportation Officials, Philadelphia, Pennsylvania, EUA (1995).

[26] AASHTO, T 19-93 - Standard test method for unit weight and voids in aggregate, American Association of State Highway and Transportation Officials, Washington, DC, EUA (1997).

[27] ASTM, C 127-88 - Standard test method for specific gravity and absorption of coarse aggregate, American Society for Testing and Materials, Philadelphia, Pennsylvania, EUA (1988).

[28] ASTM, C 128-93 - Standard test method for specific gravity and absorption of fine aggregate, American Society for Testing and Materials, Philadelphia, Pennsylvania, EUA (1993).

[29] SHRP, Superior performing asphalt pavements (Superpave): The product of SHRP asphalt research program, Superpave Series N ${ }^{\circ}$. 1, Strategic Highway Res. Program (1994).

[30] SHRP, Superior performance asphalt pavements (Superpave): The product of SHRP asphalt research program. Superpave Series $N^{\circ}$. 2, Strategic Highway Res. Program (1994).

[31] DNER, ME 085/94 - Material finamente pulverizado: Determinação da massa específica real, Departamento Nacional de Infra-Estrutura de Transportes, Rio de Janeiro, RJ (1994).

[32] DNER, ME 367/97 - Material de enchimento para misturas betuminosas, Departamento Nacional de InfraEstrutura de Transportes, Rio de Janeiro, RJ (1997).

[33] TRB, Bailey method for gradation selection in hot-mix asphalt mixture design, Transportation Research Board of the National Academies, E-Circular 044, Washington, DC, EUA (2002).

[34] J. B. Soares, F. A. Freitas, J. V. Branco, Método de previsão do teor ótimo de ligante em misturas asfálticas tipo CBUQ, 32a Reunião Anual de Pavimentação, ABPv, Vol. I, Brasília, DF (2000) pp. 148-160.

[35] DNER, ME 043/95 - Misturas betuminosas a quente: Ensaio Marshall, Departamento Nacional de Infra-Estrutura de Transportes, Rio de Janeiro, RJ (1995).

[36] AASHTO, PP-2 - Standard pratice for mixture conditioning of mix asphalt, American Association of State Highway and Transportation Officials, Washington, DC, EUA (1999).

[37]DNER, ME 004/94 - Material Betuminoso: determinação da viscosidade Saybolt-Furol a alta temperatura método da película delgada, Departamento Nacional de Infra-Estutura de Transportes, Rio de Janeiro, RJ (1994).

[38] ASTM, D 2041 - Standard test method for theoretical maximum specific gravity and density of bituminous paving mixtures, American Society for Testing and Materials, Philadelphia, Pennsylvania, EUA (2000).

[39] F. R. G. Nunes, Caracterização mecânica de misturas asfálticas com agregados sintéticos de argila calcinada quanto à deformação permanente, Diss. Mestrado, Universidade Federal do Ceará, Programa de Mestrado em Engenharia de Transportes, Fortaleza, CE (2006).

[40] F. R. G. Nunes, C. L. Silva, C. A. Frota, Transportes (Submetido), Obtenção do módulo dinâmico de misturas asfálticas confeccionadas com agregados sintéticos de argila calcinada,utilizando o ensaio de compressão diametral, Rio de Janeiro, RJ (2008).

(Rec 07/07/2008, Rev.08/04/09, Ac. 09/05/09) 